Article

\title{
Suppression of Aflatoxin Biosynthesis in Aspergillus flavus by 2-Phenylethanol Is Associated with Stimulated Growth and Decreased Degradation of Branched-Chain Amino Acids
}

\author{
Perng-Kuang Chang ${ }^{1, *}$, Sui Sheng T. Hua ${ }^{2}$, Siov Bouy L. Sarreal ${ }^{2}$ and Robert W. Li ${ }^{3}$
}

1 Southern Regional Research Center, Agricultural Research Service, U. S. Department of Agriculture, New Orleans, LA 70124, USA

2 Western Regional Research Center, Agricultural Research Service, U. S. Department of Agriculture, Albany, CA 94710, USA; E-Mails: sylvia.hua@ars.usda.gov (S.S.T.H.); siov.sarreal@ars.usda.gov (S.B.L.S.)

3 Animal Genomics and Improvement Laboratory, Agricultural Research Service,

U. S. Department of Agriculture, Beltsville, MD 20705, USA; E-Mail: robert.li@ars.usda.gov

* Author to whom correspondence should be addressed; E-Mail: perngkuang.chang@ars.usda.gov; Tel.: +1-504-286-4208; Fax: +1-504-286-4419.

Academic Editor: Jiujiang Yu

Received: 2 July 2015 / Accepted: 17 September 2015 / Published: 24 September 2015

\begin{abstract}
The saprophytic soil fungus Aspergillus flavus infects crops and produces aflatoxin. Pichia anomala, which is a biocontrol yeast and produces the major volatile 2-phenylethanol (2-PE), is able to reduce growth of $A$. flavus and aflatoxin production when applied onto pistachio trees. High levels of 2-PE are lethal to A. flavus and other fungi. However, at low levels, the underlying mechanism of 2-PE to inhibit aflatoxin production remains unclear. In this study, we characterized the temporal transcriptome response of $A$. flavus to 2-PE at a subinhibitory level $(1 \mu \mathrm{L} / \mathrm{mL})$ using RNA-Seq technology and bioinformatics tools. The treatment during the entire $72 \mathrm{~h}$ experimental period resulted in 131 of the total A. flavus 13,485 genes to be significantly impacted, of which 82 genes exhibited decreased expression. They included those encoding conidiation proteins and involved in cyclopiazonic acid biosynthesis. All genes in the aflatoxin gene cluster were also significantly decreased during the first $48 \mathrm{~h}$ treatment. Gene Ontology (GO) analyses showed that biological processes with GO terms related to catabolism of propionate and branched-chain amino acids (valine, leucine and isoleucine) were significantly enriched in the down-regulated gene group, while those associated with ribosome biogenesis, translation, and biosynthesis of $\alpha$-amino acids
\end{abstract}


were over-represented among the up-regulated genes. Kyoto Encyclopedia of Genes and Genomes (KEGG) pathway analysis revealed that metabolic pathways negatively impacted among the down-regulated genes parallel to those active at $30^{\circ} \mathrm{C}$, a condition conducive to aflatoxin biosynthesis. In contrast, metabolic pathways positively related to the up-regulated gene group resembled those at $37^{\circ} \mathrm{C}$, which favors rapid fungal growth and is inhibitory to aflatoxin biosynthesis. The results showed that 2-PE at a low level stimulated active growth of A. flavus but concomitantly rendered decreased activities in branched-chain amino acid degradation. Since secondary metabolism occurs after active growth has ceased, this growth stimulation resulted in suppression of expression of aflatoxin biosynthesis genes. On the other hand, increased activities in degradation pathways for branched-chain amino acids probably are required for the activation of the aflatoxin pathway by providing building blocks and energy regeneration. Metabolic flux in primary metabolism apparently has an important role in the expression of genes of secondary metabolism.

Keywords: Aspergillus flavus; 2-phenylethanol; aflatoxin; gene ontology; metabolic pathway; functional genomics

\section{Introduction}

Aspergillus flavus, which is a common plant and an opportunistic human pathogen, can produce the carcinogenic aflatoxin. Contamination of crops such as corn, cotton, peanuts, and tree nuts by aflatoxin poses a great food safety risk especially in developing countries due to ineffective farming practices and the lack of proper storage facilities. Aflatoxin contamination can also result in devastating economic losses because of strict regulations on dissemination of contaminated products [1]. Currently, there are no commercially available fungal cultivars resistant to the infection by $A$. flavus. The only promising intervention method showing measurable extents of control of aflatoxin contamination is to use biological control, which includes applying atoxigenic A. flavus strains, such as AF36 [2], K49 [3] or Afla-Guard ${ }^{\circledR}[4]$, to outcompete toxigenic strains in the field or spraying a yeast formulation to pistachio trees to prevent fungal growth [5]. In field tests, these biocontrol approaches have achieved greater than 80 percent reduction in aflatoxin contamination.

Pichia anomala WRL-076 is the only biocontrol yeast that has been shown to inhibit growth and aflatoxin production of $A$. flavus [6,7]. Most recently, 2-phenylethanol (2-PE) has been identified as the major volatile compound produced by this yeast [8]. 2-PE is widely found in nature, especially in flower extracts and fragrant essential oils. It has a pleasant floral odor and thus is a common ingredient of perfume. Yeast such as Candida albicans [9], Kluyveromyces marxianus [10], Saccharomyces cerevisiae [11] and Kloeckera apiculata [12] also produce 2-PE. This volatile has been demonstrated to have inhibitory properties against Penicillium italicum, which causes postharvest citrus decay [12]. The underlying mechanisms of 2-PE inhibition on growth at high concentrations have been reported on bacteria and fungi, which mainly disrupt organelles like mitochondria and nucleus, and synthesis of macromolecules, such as enzymes [13]. 
A better understanding of the mode of action of 2-PE at low concentrations, a scenario likely to be encountered in field applications of the biocontrol yeast, is critical to the development of an effective biocontrol formulation. At subinhibitory levels to fungal mycelial growth [8], how 2-PE affects aflatoxin biosynthesis is still not well understood. The objective of this study was to use the RNA-Seq approach to determine transcriptomic changes in A. flavus treated by a subinhibitory concentration $(1.0 \mu \mathrm{L} / \mathrm{mL})$ of 2-PE and to examine whether changes in the expression of specific genes of certain metabolic pathway had a bearing on inhibition of aflatoxin production. At this low concentration, 2-PE mostly stimulated fungal growth as evidenced by gene ontology $(\mathrm{GO})$ enrichment analyses showing the increased structural constituent of ribosome and an active translation ( $\alpha$-amino acid biosynthesis). The outcomes, along with a decrease in the degradation of branched-chain amino acids, were correlated with the suppression of all aflatoxin pathway gene expression.

\section{Results}

\subsection{Summary of RNA-Seq Datasets and Statistic Analysis}

The sum of single-end reads of the three biological replicates obtained from each of the experimental conditions that passed the quality control procedures ranged from 59 to 92 million (Table S1). Of the total 433 million reads, about $66.5 \%$ were mapped uniquely to the gene regions of $A$. flavus NRRL3357. Among these reads about $96.9 \%$ were located in the exon regions and $3.1 \%$ were located in the intron regions. Volcano plots derived from the $24 \mathrm{~h}, 48 \mathrm{~h}$ and $72 \mathrm{~h}$ gene expression data showing original $p$-values on the $y$-axis and fold change on the $x$-axis was generated (Figure 1). The overall fold changes at these three time points did not vary greatly, but the $p$-value range changed from E-270 at $24 \mathrm{~h}$ to E-67 at $72 \mathrm{~h}$, which indicated a decreasing trend in the significance of differentially expressed gene as cultures aged.

Statistical analyses using the "Exact Test" on the RPKM counts with the total count filter cutoff of 5 and the FDR (False Discovery Rate) correction of $p<0.05$ were performed to eliminate those false-positive genes that were initially considered positive based on original $p$-values. Table 1 summarizes the corrected numbers of differentially expressed genes obtained at a single or combined time points, which represent different growth periods. The final numbers of genes differentially expressed at these periods reflected the trend observed from the volcano plots (Table S2). Depending on the time point or the period examined, the number of differentially expressed genes based on the corrected $\mathrm{p}$-values decreased to $25 \%-50 \%$ compared to those based on the original $p$-value. Only 131 of the total 13,458 genes in the 2-PE treated cultures were differentially expressed during the entire $72 \mathrm{~h}$ period (Table 1). Clustering genes for cyclopiazonic acid biosynthesis (AFLA_139470, AFLA_139480, and AFLA_139490) and genes for conidiation proteins (AFLA_044790, AFLA_044800, and AFLA_083110) were among these significantly down-regulated during the $72 \mathrm{~h}$ period. 

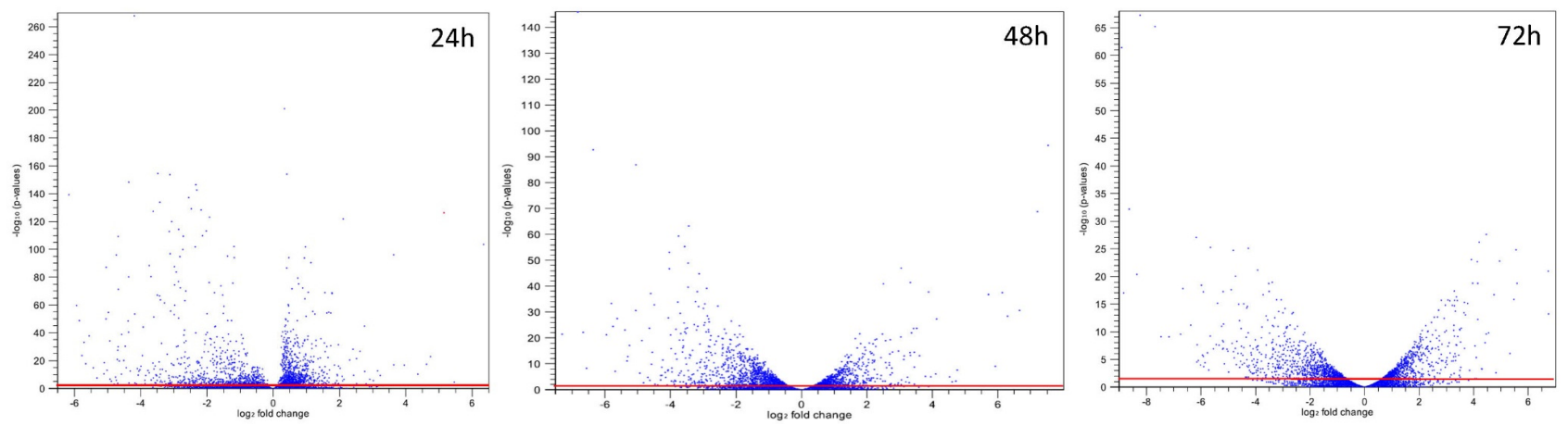

Figure 1. Volcano plots showing original $p$-values versus fold change. Each point represents the results of a gene derived from the comparison of three 2-PE treated replicate samples to those untreated samples. The $x$-axis is the $\log 2$ transformed fold change in gene expression after the 2 -PE treatment. The $y$-axis is the $-\log 10$ transformed original $p$-value. A $p$-value of 0.05 is equivalent to the value of 1.30 in the $-\log 10$ transformed $y$-axis. Genes above the (red) line are those differentially expressed $(p<0.05)$.

Table 1. Differentially expressed genes in the 2-PE treated $A$. flavus at different growth periods.

\begin{tabular}{ccccccc}
\hline \multirow{2}{*}{ Expression $^{\text {a }}$} & \multicolumn{7}{c}{ Time } \\
\cline { 2 - 7 } & $\mathbf{2 4} \mathbf{h}$ & $\mathbf{4 8} \mathbf{~ h}$ & $\mathbf{7 2} \mathbf{~ h}$ & $\mathbf{2 4} \boldsymbol{\&} \mathbf{4 8} \mathbf{~ h}$ & $\mathbf{4 8 ~ \& 7 2} \mathbf{~ h}$ & $\mathbf{2 4} \boldsymbol{\&} \mathbf{4 8} \boldsymbol{\&} \mathbf{7 2} \mathbf{~ h}$ \\
\hline Decreased & 967 & 959 & 671 & 255 & 291 & 82 \\
Increased & 1200 & 593 & 527 & 163 & 197 & 49 \\
\hline
\end{tabular}

a: FDR corrected $p$-value is $<0.05$.

\subsection{Treatment by 2-PE Decreased Expression of All Genes in the Aflatoxin Gene Cluster of A. flavus}

For a better understanding of the effect of 2-PE on the aflatoxin gene expression by $A$. flavus, all genes in the aflatoxin biosynthesis gene cluster were examined (Table 2). The decrease in gene expression was most prominent at $24 \mathrm{~h}$ and ranged from two- to greater than 18-fold. In comparison, the decrease at $48 \mathrm{~h}$ and $72 \mathrm{~h}$ was within three-fold. A. flavus can only produce aflatoxin $\mathrm{B}_{1}$ and $\mathrm{B}_{2}$. The expression pattern of $\operatorname{nor} B$ and $\operatorname{cyp} A$, which are involved in the biosynthesis of aflatoxin $\mathrm{G}_{1}$ and $\mathrm{G}_{2}$, were less correlated with that of other genes responsible for B-type aflatoxin production. Although fold of decreases at $48 \mathrm{~h}$ and $72 \mathrm{~h}$ were comparable, all values at $72 \mathrm{~h}$ after the FDR correction were not significant (FDR corrected $p$-values $>0.05$, Table 2 ). This was found to be caused by increased variance for each gene at $72 \mathrm{~h}$ compared to its counterpart at $24 \mathrm{~h}$ and $48 \mathrm{~h}$, respectively. 
Table 2. Fold change in expression of genes in the aflatoxin gene cluster.

\begin{tabular}{|c|c|c|c|c|c|c|c|}
\hline Gene ID & Gene Name and Product & $24 \mathrm{~h}$ & FDR & $48 \mathrm{~h}$ & FDR & $72 \mathrm{~h}$ & FDR \\
\hline AFLA_139140 & aflYa/nadA/NADH oxidase & $-5.91^{\text {a }}$ & 1.00 & -1.89 & 0.00 & $-2.67^{\mathrm{a}}$ & 0.07 \\
\hline AFLA_139150 & aflY/hypA/hypP/hypothetical protein & -10.32 & 0.00 & -2.39 & 0.00 & $-2.17^{\mathrm{a}}$ & 0.30 \\
\hline AFLA_139160 & aflX/ordB/monooxygenase/oxidase & -7.33 & 0.00 & -1.56 & 0.00 & $-2.07^{\mathrm{a}}$ & 0.31 \\
\hline AFLA_139170 & aflW/mox $Y /$ monooxygenase & -8.36 & 0.00 & -2.05 & 0.00 & $-2.09^{\mathrm{a}}$ & 0.35 \\
\hline AFLA_139180 & aflV/cyp X/P450 monooxygenase & -11.59 & 0.00 & -2.12 & 0.00 & $-2.03^{\mathrm{a}}$ & 0.40 \\
\hline AFLA_139190 & $a f l K / v b s /$ VERB synthase & -5.67 & 0.00 & -2.98 & 0.00 & $-2.50^{\mathrm{a}}$ & 0.14 \\
\hline AFLA_139200 & aflQ/ordA/P450 monooxygenase & $-10.57^{\mathrm{a}}$ & 0.07 & -1.87 & 0.00 & $-2.11^{\mathrm{a}}$ & 0.29 \\
\hline AFLA_139210 & aflP/omtA/omt 1/O-methyltransferase A & -14.69 & 0.00 & -2.27 & 0.00 & $-2.03^{\mathrm{a}}$ & 0.41 \\
\hline AFLA_139220 & aflO/omtB/dmtA/O-methyltransferase B & -5.83 & 0.00 & -1.45 & 0.03 & $-1.62^{\mathrm{a}}$ & 0.74 \\
\hline AFLA_139230 & aflI/avfA/P450 monooxygenase & -21.13 & 0.02 & -2.28 & 0.00 & $-2.11^{\mathrm{a}}$ & 0.21 \\
\hline AFLA_139240 & aflLa/hypB/hypothetical protein & -13.51 & 0.00 & -2.46 & 0.00 & $-2.37^{\mathrm{a}}$ & 0.16 \\
\hline AFLA_139250 & aflL/verB/desaturase/P450 monooxygenase & -8.64 & 0.00 & -2.44 & 0.00 & $-2.36^{\mathrm{a}}$ & 0.15 \\
\hline AFLA_139260 & aflG/avnA/ord1/P450 monooxygenase & $-5.09^{\mathrm{a}}$ & 0.19 & -1.74 & 0.00 & $-2.17^{\mathrm{a}}$ & 0.20 \\
\hline AFLA_139270 & aflNa/hypD/hypothetical protein & -3.32 & 0.00 & $-1.09^{\mathrm{a}}$ & 0.34 & $-1.76^{\mathrm{a}}$ & 0.46 \\
\hline AFLA_139280 & aflN/verA/monooxygenase & $-4.29^{\mathrm{a}}$ & 0.11 & -1.58 & 0.00 & $-2.27^{\mathrm{a}}$ & 0.18 \\
\hline AFLA_139290 & aflMa/hypE/hypothetical protein & -6.94 & 0.00 & -1.41 & 0.01 & $-1.74^{\mathrm{a}}$ & 0.56 \\
\hline AFLA_139300 & aflM/ver $1 /$ dehydrogenase/ketoreductase & -18.07 & 0.00 & -1.79 & 0.00 & $-1.83^{\mathrm{a}}$ & 0.55 \\
\hline AFLA_139310 & aflE/norA/aad/adh2/NOR reductase & -10.11 & 0.00 & -1.86 & 0.00 & $-2.32^{\mathrm{a}}$ & 0.19 \\
\hline AFLA_139320 & aflJ/estA/esterase & -4.95 & 0.00 & -1.42 & 0.01 & $-2.15^{\mathrm{a}}$ & 0.29 \\
\hline AFLA_139330 & aflH/adhA/short chain alcohol dehydrogenase & -4.75 & 0.00 & -1.78 & 0.00 & $-2.22^{\mathrm{a}}$ & 0.22 \\
\hline AFLA_139340 & aflS/aflJ/pathway regulator & -2.67 & 0.00 & $-1.12^{\mathrm{a}}$ & 0.25 & $-1.10^{\mathrm{a}}$ & 1.00 \\
\hline AFLA_139360 & aflR/apa 2/afl2/C6 transcription factor & -2.00 & 0.02 & $-1.10^{\mathrm{a}}$ & 0.43 & $-1.40^{\mathrm{a}}$ & 0.72 \\
\hline AFLA_139370 & aflB/fasl/fatty acid synthase beta subunit & $-2.54^{\mathrm{a}}$ & 0.24 & -1.79 & 0.00 & $-1.58^{\mathrm{a}}$ & 0.52 \\
\hline
\end{tabular}


Table 2. Cont.

\begin{tabular}{|c|c|c|c|c|c|c|c|}
\hline Gene ID & Gene Name and Product & $24 \mathrm{~h}$ & FDR & $48 \mathrm{~h}$ & FDR & $72 \mathrm{~h}$ & FDR \\
\hline AFLA_139380 & aflA/fas $2 /$ hexA/fatty acid synthase alpha subunit & $-1.27^{\mathrm{a}}$ & 1.00 & -2.13 & 0.00 & $-2.29^{\mathrm{a}}$ & 0.09 \\
\hline AFLA_139390 & aflD/nor $1 /$ reductase & -3.46 & 0.00 & -2.33 & 0.00 & $-2.29^{\mathrm{a}}$ & 0.21 \\
\hline AFLA_139400 & aflCa/hypC/hypothetical protein & -4.53 & 0.00 & -2.60 & 0.00 & $-2.66^{\mathrm{a}}$ & 0.08 \\
\hline AFLA_139410 & aflC/pksA/pksL1/polyketide synthase & $-2.01^{\mathrm{a}}$ & 0.59 & -2.32 & 0.00 & $-2.57^{\mathrm{a}}$ & 0.12 \\
\hline AFLA_139420 & aflT/transmembrane protein & $-5.12^{\mathrm{a}}$ & 0.62 & -1.53 & 0.00 & $-1.79^{\mathrm{a}}$ & 0.15 \\
\hline AFLA_139430 & aflU/cypA/P450 monooxygenase & $-4.92^{\mathrm{a}}$ & 1.00 & $-1.27^{\mathrm{a}}$ & 0.59 & $-1.29^{\mathrm{a}}$ & 1.00 \\
\hline AFLA_139440 & aflF/norB/dehydrogenase & $-7.48^{\mathrm{a}}$ & 1.00 & $-1.18^{\mathrm{a}}$ & 0.71 & $-1.26^{\mathrm{a}}$ & 1.00 \\
\hline
\end{tabular}

a: The FDR $>0.05$. 


\subsection{GO Functional Classification of Differentially Expressed Genes}

In light of the enlarged variance for the aflatoxin gene expression level at the late stage of growth, we arbitrarily selected $48 \mathrm{~h}$ as the cut-off time for evaluating 2-PE treatment on GO functional categories of the differentially expressed genes. The treatment resulted in 255 and 163 genes that were down- and up-regulated, respectively (Table 1). GO functional analyses of the down-regulated genes performed at level 2 showed that cell (GO:0005623), membrane (GO:0016020) and organelle GO:0043226) were the GO terms associated with the cellular component category, metabolic process (GO:0008152), singleorganism process (GO:0044699) and cellular process (GO:0009987) were associated with the biological process category, and catalytic activity (GO:0003824) and binding (GO:0005488) were associated with the molecular function category (Figure 2). Similarly, the up-regulated genes with these GO functional classifications were also predominant (Table S3).
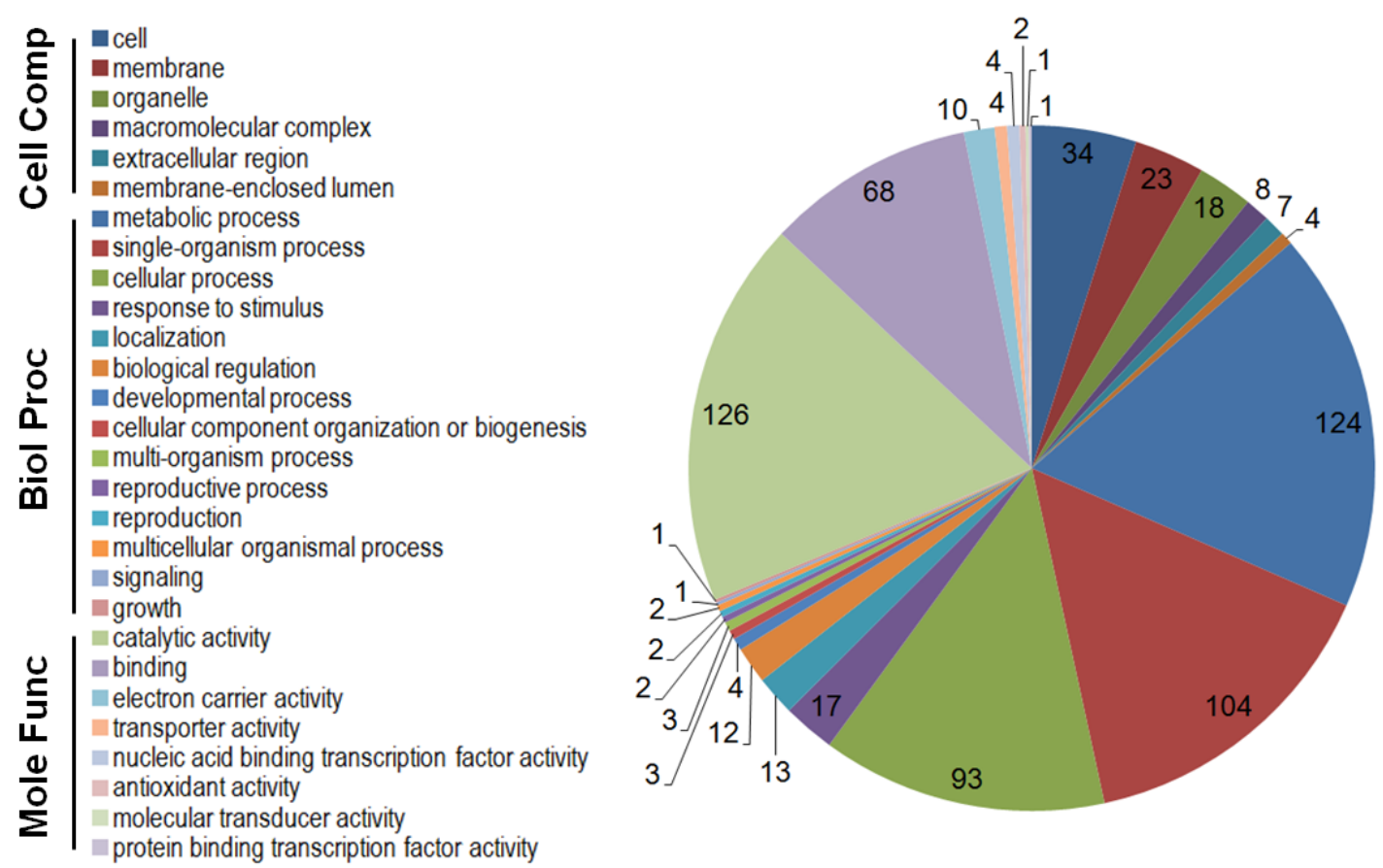

Figure 2. GO categories and GO terms of the 255 significantly down-regulated genes during the first $48 \mathrm{~h}$ growth period. The three categories are cellular component, biological process and molecular function. The combined graphic was generated using the GO level 2.

\subsection{GO Enrichment and KEGG Metabolic Pathway Analysis of the Differentially Expressed Genes}

The specific GO terms enriched in the down-regulated gene group were mainly in the biological process category. These included catabolism of branched-chain amino acids of valine, leucine and isoleucine, and in the biosynthetic pathway of polyketide-derived sterigmatocystin and aflatoxin (Figure 3a). The specific GO terms enriched in the up-regulated gene group (Figure $3 b$ ) in the cellar component category were specific for the cytosolic small ribosomal subunit. The molecular function was mainly responsible for the structural constituent of the ribosome, i.e., the integrity of ribosome. In the biological processes category, the GO terms were primarily for the production of mature rRNA, which include endonucleolytic cleavage in ITS1 to separate SSU (small subunit)-rRNA from 5.8S rRNA and LSU (large subunit)-rRNA 
from tricistronic rRNA transcript (SSU-rRNA, 5.8S rRNA, LSU-rRNA), endonucleolytic cleavage to generate mature 3'-end of SSU-rRNA from (SSU-rRNA, 5.8S rRNA, LSU-rRNA), translation, and biosynthesis (anabolism) of $\alpha$-amino acids. Tables S4 and S5 further show the summaries of the general (detailed) GO terms of the down- and up-regulated genes including IDs, respective categories, FDRs and $p$-values.

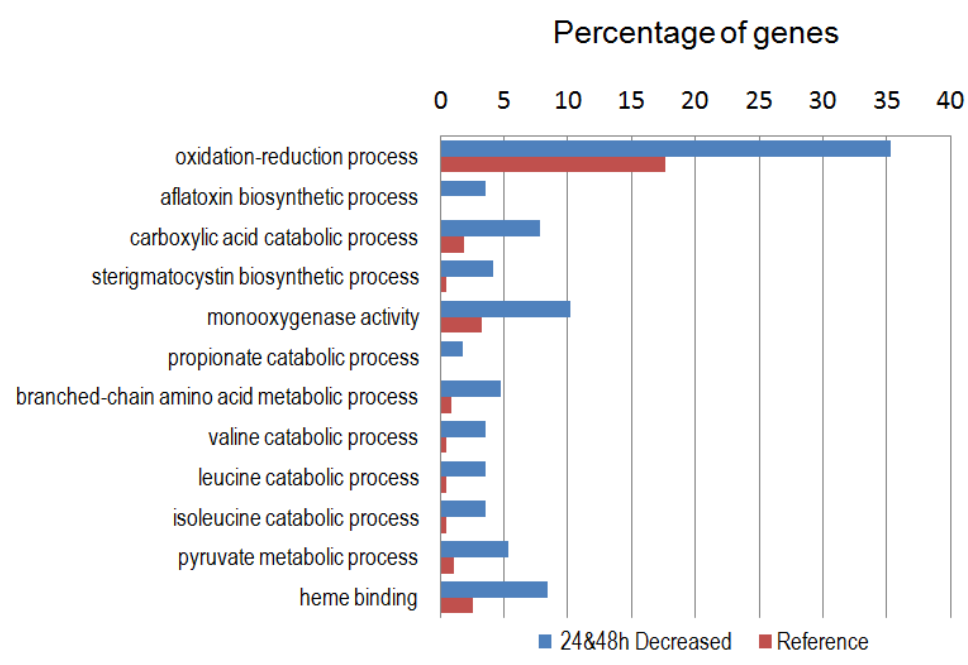

(a)

Percentage of genes

$\begin{array}{llllllll}0 & 5 & 10 & 15 & 20 & 25 & 30 & 35\end{array}$

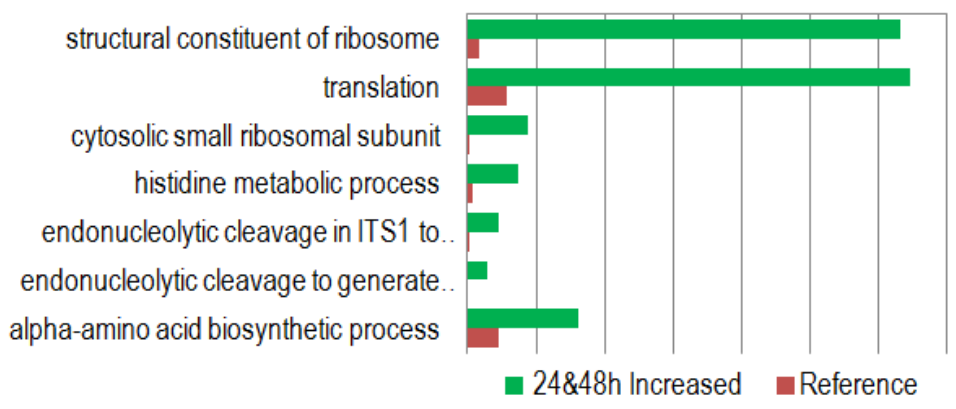

(b)

Figure 3. Functional enrichment analyses of differentially expressed genes: (a) The 255 significantly down-regulated genes. The propionate catabolic process is the 2-methylcitrate cycle. (b) The 163 significantly up-regulated genes. Endonucleolytic cleavage: see Section 2.4 for full description. Fisher's Exact Test with the FDR corrected $p$-value of $<0.05$ was used. The reference gene set contained the remaining genes with GO annotations, that is, the whole genes with GO annotations minus the down- or the up-regulated genes with GO annotations.

The KEGG pathway analysis confirmed that the amino acid metabolic pathways identified in the down-regulated gene group were consistent with the enriched GO terms in the biological processes category including those involved in metabolism branched-chain amino acids (Table 3). In addition, other pathways involved in the metabolism of amino acids with non-polar (serine and threonine) and polar side chain (tryptophan, phenylalanine and glycine) were identified. However, the metabolic 
pathways associated with the up-regulated gene group were those for basic (histidine, arginine and proline) and acidic (aspartate and glutamate) amino acids.

Table 3. KEGG pathways of differentially expressed genes with GO terms enriched.

\begin{tabular}{ccc}
\hline KEGG Metabolic Pathway a & \#Seq/\#Enz & Order of Abundance $^{\text {b }}$ \\
\hline Down-Regulated Gene Group & \\
\hline Pyruvate metabolism & $7 / 8$ & 18 \\
Valine, leucine and isoleucine degradation & $7 / 6$ & 32 \\
Propanoate metabolism & $6 / 5$ & 33 \\
Tryptophan metabolism & $5 / 4$ & 7 \\
Glyoxylate and dicarboxylate metabolism & $5 / 4$ & 28 \\
Phenylalanine metabolism & $4 / 3$ & 8 \\
Fatty acid degradation & $4 / 4$ & 10 \\
B-Alanine metabolism & $4 / 3$ & 3 \\
Ulycine, serine and threonine metabolism & $4 / 4$ & 48 \\
\hline Histidine metabolism & $4 / 6$ & 53 \\
Arginine and proline metabolism & $3 / 5$ & 5 \\
Pyruvate metabolism & $3 / 2$ & 18 \\
Citrate cycle (TCA cycle) & $2 / 1$ & 42 \\
\hline Alanine, aspartate and glutamate metabolism & $2 / 2$ & 21 \\
Phenylalanine, tyrosine and tryptophan biosynthesis & $2 / 5$ & 44 \\
\hline a: 74 and 59 GO-enriched genes in the down- and up-regulated gene groups were used, respectively; \\
b: The ordered rank of gene abundance of all 129 KEGG pathways of A. flavus [14].
\end{tabular}

\section{Discussion}

2-PE at high concentrations $(0.3 \%-0.5 \%)$ is lethal to bacteria and fungi $[8,15-18]$. At a sublethal level, 2-PE delays spore germination and mycelial growth of $A$. flavus. Previous results indicate that 2-PE $(1.0 \mu \mathrm{L} / \mathrm{mL})$ used in the present study has no discernible adverse effects on fungal growth and does not disrupt membrane integrity [8]. Nonetheless, 2-PE at this concentration inhibits the expression of all genes in the aflatoxin gene cluster (Table 2) and the cyclopiazonic acid gene cluster. The marked decrease in the expression of the majority of aflatoxin genes within the first $24 \mathrm{~h}$ growth period correlates well with the two- to three-fold decrease in the pathway regulatory genes of aflR and aflJ, which are required for transcriptional activation of other aflatoxin biosynthetic genes [19,20]. The smaller decrease in the aflR and aflJ expression at $48 \mathrm{~h}$ and $72 \mathrm{~h}$ also correlates with a lesser extent of decrease in other gene expression. Global regulatory genes such as $v e A$ and laeA control the expression of aflR and aflJ [21,22]. Although the transcriptomic data show a slight decrease $(<1.5$-fold $)$ in the expression of both of veA and laeA genes, the decrease is not significant (Table S6). The decrease in all aflatoxin gene expression in the treated $A$. flavus likely results from a broad change in physiology and metabolism (see below).

The GO enrichment analysis of the down-regulated gene group shows that GO terms associated with biosynthesis of toxic secondary metabolites (aflatoxin and sterigmatocystin), and catabolism of carboxylic acid and branched-chain amino acids (valine, leucine and isoleucine) are over-represented. These results 
are indicative of a metabolic flux being channeled toward primary metabolism for synthesis of $C$ - and $N$-containing macromolecules. In the up-regulated gene group GO terms associated with ribosome synthesis, translation and anabolism of $\alpha$-amino acids are enriched. Taken together, the 2-PE treatment promotes active fungal growth. The KEGG metabolic pathway analyses of the GO-enriched down- and up-regulated gene groups also support this explanation (Table 3). Although the pathway of pyruvate metabolism was identified in both down- and up-regulated gene groups, it suggests that pyruvate enters the mitochondrion and metabolized further for acetyl-CoA production. Acetyl-CoA then either enter the citric acid (TCA) cycle to the processes of energy generation or enter biosynthetic pathways for cell growth. The optimal temperature for aflatoxin biosynthesis is $30{ }^{\circ} \mathrm{C}$. In contrast, a higher temperature such as $37{ }^{\circ} \mathrm{C}$ favors fungal growth but suppresses aflatoxin gene expression [23]. Previous KEGG pathway analyses indicate that growth at $30{ }^{\circ} \mathrm{C}$ favors degradation of propionate, fatty acid, valine, leucine, isoleucine, tryptophan and phenylalanine [14]. Coincidently, the 2-PE treatment, which decreases the expression of all aflatoxin biosynthesis genes, also results in decreased expression of genes in these metabolic pathways that are active at $30{ }^{\circ} \mathrm{C}$ (Table 3). The degradation of isoleucine and valine leads to the production of propionate, which is toxic to cells and inhibits cell growth [24]. Although the methylcitrate cycle is a pathway that can metabolize propionate into pyruvate, which then is used as a energy or carbon source for other metabolism [25], the expression of genes of this pathway is also downregulated (Figure 3A and Table 3- propanoate metabolism). Noticeably, the expression of genes involved in histidine metabolism, arginine and proline metabolism, alanine, aspartate and glutamate metabolism, and phenylalanine, tyrosine and tryptophan biosynthesis is up-regulated in the 2-PE treated A. flavus. The expression of genes in these four pathways is also up-regulated when A. flavus is grown at $37{ }^{\circ} \mathrm{C}$ [14]. The role of amino acid metabolism in aflatoxin biosynthesis is complex. Specific amino acids used as a sole nitrogen or carbon source have different effects on growth and aflatoxin production. Phenylalanine, tyrosine, tryptophan, proline, and asparagine are readily incorporated into aflatoxin in A. flavus [26]. The latter two amino acids also increase aflatoxin production [27]. Asparagine is essential for aflatoxin production in Aspergillus parasiticus, and it can be replaced by aspartate or alanine [28]. As a supplement, tryptophan $(50 \mathrm{mM})$ in aflatoxin-conducive medium significantly reduced aflatoxin production in A. flavus, but tyrosine at the same concentration significantly increases aflatoxin production [29]. In this study, higher activities in the catabolism of non-polar branched-chain amino acids are correlated with active aflatoxin biosynthesis, while metabolism of other basic and acidic amino acids and biosynthesis (anabolism) of non-polar side-chain tryptophan and phenylalanine are associated mainly with fungal growth (Table 3 ). These results imply that amino acids play different roles in primary metabolism and secondary metabolism.

The mode of action of 2-PE includes inhibition of synthesis of DNA [13,15,30] and disruption of membrane integrity [31,32]. Most recently Liu et al. (2014) have reported that a short $2 \mathrm{~h}$ exposure of Penicillium italicum to a lethal concentration of 2-PE $(1.5 \mu \mathrm{L} / \mathrm{mL})$ induces subcellular changes in hyphae characteristics by vacuolation, degradation of organelles, and leakage in the outer membrane of mitochondria. In the corresponding transcriptomic analysis, the expression of genes associated with ribosome and amino acid biosynthesis is down-regulated and they conclude that inhibition of the amino acids and protein biosynthesis is the cause of the lethal effect of 2-PE [12]. In this study, we show that a subinhibitory concentration of 2-PE to mycelial growth elicits in A. flavus totally opposite changes; it promotes protein synthesis for growth as evidenced by the GO enrichment analysis and the result that 
expression of about 70\% (46/67) of genes encoding known or putative ribosomal proteins is increased significantly in the first $48 \mathrm{~h}$ (FDR corrected $p$-value $<0.05$, data not shown), but it inhibits secondary metabolism, like the decreased expression of all genes in the aflatoxin and cyplopiazonic acid gene clusters. Studies have shown that plant volatiles of aldehydes, ketones, and alcohols from cotton leaf [33], corn [34], and soybean [35] either are inhibitory to fungal growth or are lethal, and some appear to inhibit aflatoxin production. Although morphological and subcellular changes are usually associated with growth inhibitory effects of these volatiles, the underlying mechanism(s) of how aflatoxin production of A. flavus or closely related A. parasiticus is decreased or abolished when exposed to sublethal levels of volatile(s) remains unclear. Nonetheless, this study shows that active growth and decreased activities in the metabolism of specific amino acids have a bearing on the decrease in all aflatoxin gene expression in the 2-PE treated A. flavus.

In the previous transcriptomic study of $A$. flavus treated by decanal [36], the con (conidiation) genes were found not to be conidia-specific but are associated with stresses because they are highly expressed in sclerotia, which are hardened hyphal aggregates for survival at unfavorable environments. In addition, Con proteins contain a characteristic stress-response KGG repeat motif. Consistently, con transcripts are not detected in growing mycelia in Neurospora crassa [37]. The greatly decreased expression in the con genes in the 2-PE treated A. flavus (Table S6) suggests that 2-PE at this low concentration $(1 \mu \mathrm{L} / \mathrm{mL})$ does not render a stress to the fungal cells. Conidiophorogenesis represents the first phase of Aspergillus development after the cessation of vegetative growth. The $\operatorname{brlA}$ gene which encodes a $\mathrm{C}_{2} \mathrm{H}_{2}$ type transcription factor controls the formation of conidiophore [38]. A delay or reduction in the $\operatorname{brlA}$ expression has been associated with the halting of programmed conidiation $[39,40]$. The brlA expression in the 2-PE treated A. flavus is four- to 10-fold lower than that of controls (Table S6), which suggests that the fungus still is at an active vegetative stage. The initiation of aflatoxin biosynthesis, a secondary metabolism, occurs when active growth slows down [41]. These observations give further support to the proposition that decreased aflatoxin gene expression in A. flavus mainly results from the stimulating effect by the subinhibitory concentration of 2-PE on fungal growth.

\section{Experimental Section}

\subsection{Fungal Strain, Medium and Culture Growth}

Aspergillus flavus NRRL3357 was maintained on Potato Dextrose Agar (PDA, Becton Dickinson, Franklin Lakes, NJ, USA). A fresh spore suspension was prepared in a $0.05 \%$ Tween 80 solution. The NYDB growth medium consisted of nutrient broth $8 \mathrm{~g}$, yeast extract $5 \mathrm{~g}$, and glucose $10 \mathrm{~g} / \mathrm{L}$. Aliquots of the spore suspension were inoculated into $20 \mathrm{~mL}$ of NYDB in a $125 \mathrm{~mL}$ flask to a final concentration of $10^{5} / \mathrm{mL}$. For the treatment set, 2-phenylethanol (2-PE, Sigma-Aldrich, St. Louis, MO, USA) was added to a final concentration of $1 \mu \mathrm{L} / \mathrm{mL}$. For the control and treatment sets three cultures of each were grown at $28^{\circ} \mathrm{C}$ on a rotary shaker at $150 \mathrm{rpm}$. At $24 \mathrm{~h}, 48 \mathrm{~h}$ and $72 \mathrm{~h}$ after inoculation, mycelia were harvested, rinsed with cold DEPC-treated water $(0.1 \%$ solution $)$, dried by vacuum suction, and ground in a chilled mortar with liquid nitrogen until a fine powder was achieved. 


\subsection{Preparation of Total RNA and Sequencing}

Total RNA isolation was carried out using RNeasy Plant Mini Kit (Qiagen, Valencia, CA, USA). The RNA samples were treated with Ambion TURBO DNA-free DNase (Ambion, Austin, TX, USA). The purity and concentrations of RNA were examined by a ND-1000 Spectrophotometer (NanoDrop Technologies, Wilmington, Delaware, DE, USA). RNA quality was assessed with an Agilent 2100 Bioanalyzer (Agilent Technologies, Santa Clara, CA, USA) and all were with RIN (RNA Integrity Number) between 6.9 and 7.8. Samples were stored in a $-80{ }^{\circ} \mathrm{C}$ freezer until use. Total RNA was processed using an Illumina TruSeq RNA Sample Prep kit, following the manufacturer's instruction (Illumina, San Diego, CA, USA). After various quality control procedures, individual RNA-Seq libraries were pooled at an equal molar ratio based on their respective sample-specific 6-bp adaptors. Pooled RNA-Seq libraries were then sequenced at $50 \mathrm{bp} /$ sequence read using an Illumina HiSeq 2000 sequencer as described previously [42]. Raw single-end sequence reads generated were filtered to remove artificial reads, adapters and low quality reads using the Illumina pipeline to generate fastq files. A total of 18 samples (two groups, three time points, and three biological replicates) were sequenced for this study. The filtered sequence reads were deposited to the NCBI Sequence Read Archive under the accession number of SRP056528 and publically accessible.

\subsection{Mapping Reads to A. flavus Reference Genome and Normalized Gene Expression Levels}

Mapping at $80 \%$ identity fraction and $80 \%$ length fraction using the RNA-Seq module of CLC Genomic Workbench version 8 was performed [43]. All reads were mapped to gene regions of $A$. flavus NRRL3357 [44] and expression values for every gene in the RPKM (Reads Per Kilobase exon model per Million mapped reads) unit [45] were calculated. These values were normalized for total exon length and the total number of matches in an experiment to allow for cross-sample comparisons.

\subsection{Statistical Analysis of Digital Gene Expression}

The statistical analysis was performed using the Empirical Analysis of DGE (Digital Gene Expression) function of CLC Genomics Workbench, which implements the "Exact Test" for two-group comparisons [46]. This method is similar to Fisher's Exact Test but takes into account overdispersion caused by biological variability. In other words, the "Exact Test" compares the counts in one set of count samples, i.e., sample replicates, against those in another set of count samples. In comparison, Fisher's Exact Test compares the counts in one sample against those of another. Total count filter cutoff number was set 5. FDR (False Discovery Rate) corrected- $p$ values were calculated from the original $p$-values [47]. FDR is the proportion of false positives among all those positive. In this study, 5\% of FDR corrected $p$-values was set to be false-positive $(p<0.05)$.

\subsection{Gene Ontology, Functional Enrichment, and KEGG Metabolic Pathways}

Blast2GO [48] was used to assign GO terms to genes differentially expressed at the first $48 \mathrm{~h}$. Functional enrichment analyses were performed on the down-and up-regulated gene groups, which were compared to the remaining genes of the whole genome using Fisher's Exact Test with Multiple Test Correction of False Discovery Rate at the threshold of 0.05. Genes associated with the enriched GO 
terms in the down- and up-regulated gene groups were also analyzed using the reference metabolic pathways of the Kyoto Encyclopedia of Genes and Genomes (KEGG) database [49].

\section{Conclusions}

Our functional genomics study shows that the inhibition of aflatoxin production by the low level of 2-PE results from its effect on promoting active growth of $A$. flavus. Metabolism of different amino acids in primary metabolism and secondary metabolism are associated with $A$. flavus growth, development, and aflatoxin production. Noticeably, aflatoxin production requires a higher activity in the catabolism of branched-chain amino acids. Likely, the end products of this degradation pathway such as acetate and propanoate not only serve as precursors that are channeled into aflatoxin biosynthesis but are also used for energy regeneration. Metabolic flux from primary metabolism can impact the expression of genes of secondary metabolism.

\section{Supplementary Materials}

Supplementary materials can be accessed at: http://www.mdpi.com/2072-6651/7/10/3887/s1.

\section{Acknowledgments}

The authors thank Leslie L. Scharfenstein for submitting RNA-Seq reads to the NCBI Sequence Read Archive.

\section{Author Contributions}

P.-K.C. and S.S.T.H. conceived and designed the experiments; S.B.L.S. and R.W.L. performed the experiments; and P.-K.C. and R.W.L. analyzed the data and wrote the paper.

\section{Conflicts of Interest}

The authors declare no conflict of interest.

\section{References}

1. Liu, Y.; Wu, F. Global burden of aflatoxin-induced hepatocellular carcinoma: A risk assessment. Environ. Health Perspect. 2010, 118, 818-824.

2. Cotty, P.J. Influence of field application of an atoxigenic strain of Aspergillus flavus on the populations of $A$. flavus infecting cotton bolls and on the aflatoxin content of cottonseed. Phytopathology 1994, 84, 1270-1277.

3. Abbas, H.K.; Zablotowicz, R.M.; Horn, B.W.; Phillips, N.A.; Johnson, B.J.; Jin, X.; Abel, C.A. Comparison of major biocontrol strains of non-aflatoxigenic Aspergillus flavus for the reduction of aflatoxins and cyclopiazonic acid in maize. Food Addit. Contam. 2011, 28, 198-208.

4. Dorner, J.W. Development of biocontrol technology to manage aflatoxin contamination in peanuts. Peanut Sci. 2009, 36, 60-67. 
5. Hua, S.S. Progress in prevention of aflatoxin contamination in food by preharvest application of Pichia anomala WRL-076. In Recent Advances in Multidisciplinary Applied Microbiology; Mendez-Vilas, A., Ed.; Wiley-VCH: Weinheim, Germany, 2006; pp. 322-326.

6. Hua, S.S.; Baker, J.L.; Flores-Espiritu, M. Interactions of saprophytic yeasts with a nor mutant of Aspergillus flavus. Appl. Environ. Microbiol. 1999, 65, 2738-2740.

7. Hua, S.S.; Brandl, M.T.; Hernlem, B.; Eng, J.G.; Sarreal, S.B. Fluorescent viability stains to probe the metabolic status of aflatoxigenic fungus in dual culture of Aspergillus flavus and Pichia anomala. Mycopathologia 2011, 171, 133-138.

8. Hua, S.S.; Beck, J.J.; Sarreal, S.B.; Gee, W. The major volatile compound 2-phenylethanol from the biocontrol yeast, Pichia anomala, inhibits growth and expression of aflatoxin biosynthetic genes of Aspergillus flavus. Mycotoxin Res. 2015, 30, 71-78.

9. Lingappa, B.T.; Prasad, M.; Lingappa, Y.; Hunt, D.F.; Biemann, K. Phenethyl alcohol and tryptophol: Autoantibiotics produced by the fungus Candida albicans. Science N.Y. 1969, 163, 192-194.

10. Gao, F.; Daugulis, A.J. Bioproduction of the aroma compound 2-phenylethanol in a solid-liquid two-phase partitioning bioreactor system by Kluyveromyces marxianus. Biotechnol. Bioeng. 2009, 104, 332-339.

11. Wang, H.; Dong, Q.; Meng, C.; Shi, X.A.; Guo, Y. A continuous and adsorptive bioprocess for efficient production of the natural aroma chemical 2-phenylethanol with yeast. Enzyme Microb. Technol. 2011, 48, 404-407.

12. Liu, P.; Cheng, Y.; Yang, M.; Liu, Y.; Chen, K.; Long, C.A.; Deng, X. Mechanisms of action for 2-phenylethanol isolated from Kloeckera apiculata in control of Penicillium molds of citrus fruits. BMC Microbiol. 2014, 14, 242.

13. Rosenkranz, H.S.; Carr, H.S.; Rose, H.M. Phenethyl alcohol. I. Effect on macromolecular synthesis of Escherichia coli. J. Bacteriol. 1965, 89, 1354-1369.

14. Chang, P.-K.; Scharfenstein, L.L. Aspergillus flavus Blast2GO gene ontology database: Elevated growth temperature alters amino acid metabolism. Genet. Genome Res. 2014, 1, 1.

15. Berrah, G.; Konetzka, W.A. Selective and reversible inhibition of the synthesis of bacterial deoxyribonucleic acid by phenethyl alcohol. J. Bacteriol. 1962, 83, 738-744.

16. Lester, G. Inhibition of growth, synthesis, and permeability in Neurospora crassa by phenethyl alcohol. J. Bacteriol. 1965, 90, 29-37.

17. Fraud, S.; Rees, E.L.; Mahenthiralingam, E.; Russell, A.D.; Maillard, J.Y. Aromatic alcohols and their effect on Gram-negative bacteria, cocci and mycobacteria. J. Antimicrob. Chemother. 2003, $51,1435-1436$.

18. Rosenkranz, H.S.; Carr, H.S.; Rose, H.M. Phenethyl alcohol. II. Effect on thymine-requiring Escherichia coli. J. Bacteriol. 1965, 89, 1370-1373.

19. Chang, P.-K. The Aspergillus parasiticus protein AFLJ interacts with the aflatoxin pathway-specific regulator AFLR. Mol. Genet. Genom. 2003, 268, 711-719.

20. Chang, P.-K.; Ehrlich, K.C.; Yu, J.; Bhatnagar, D.; Cleveland, T.E. Increased expression of Aspergillus parasiticus aflR, encoding a sequence-specific DNA-binding protein, relieves nitrate inhibition of aflatoxin biosynthesis. Appl. Environ. Microbiol. 1995, 61, 2372-2377. 
21. Calvo, A.M.; Bok, J.; Brooks, W.; Keller, N.P. veA is required for toxin and sclerotial production in Aspergillus parasiticus. Appl. Environ. Microbiol. 2004, 70, 4733-4739.

22. Chang, P.-K.; Scharfenstein, L.L.; Ehrlich, K.C.; Wei, Q.; Bhatnagar, D.; Ingber, B.F. Effects of laeA deletion on Aspergillus flavus conidial development and hydrophobicity may contribute to loss of aflatoxin production. Fungal Biol. 2012, 116, 298-307.

23. Yu, J.; Fedorova, N.D.; Montalbano, B.G.; Bhatnagar, D.; Cleveland, T.E.; Bennett, J.W.; Nierman, W.C. Tight control of mycotoxin biosynthesis gene expression in Aspergillus flavus by temperature as revealed by RNA-Seq. FEMS Microbiol. Lett. 2011, 322, 145-149.

24. Brock, M. Generation and phenotypic characterization of Aspergillus nidulans methylisocitrate lyase deletion mutants: Methylisocitrate inhibits growth and conidiation. Appl. Environ. Microbiol. 2005, 71, 5465-5475.

25. Upton, A.M.; McKinney, J.D. Role of the methylcitrate cycle in propionate metabolism and detoxification in Mycobacterium smegmatis. Microbiology Read. Engl. 2007, 153, 3973-3982.

26. Adye, J.; Mateles, R.I. Incorporation of labelled compounds into aflatoxins. Biochim. Biophys. Acta 1964, 86, 418-420.

27. Payne, G.A.; Hagler, W.M., Jr. Effect of specific amino acids on growth and aflatoxin production by Aspergillus parasiticus and Aspergillus flavus in defined media. Appl. Environ. Microbiol. 1983, 46, 805-812.

28. Reddy, T.V.; Viswanathan, L.; Venkitasubramanian, T.A. Factors affecting aflatoxin production by Aspergillus parasiticus in a chemically defined medium. J. Gen. Microbiol. 1979, 114, 409-413.

29. Wilkinson, J.R.; Yu, J.; Bland, J.M.; Nierman, W.C.; Bhatnagar, D.; Cleveland, T.E. Amino acid supplementation reveals differential regulation of aflatoxin biosynthesis in Aspergillus flavus NRRL 3357 and Aspergillus parasiticus SRRC 143. Appl. Microbiol. Biotechnol. 2007, 74, 1308-1319.

30. Treick, R.W.; Konetzka, W.A. Physiological state of Escherichia coli and the inhibition of deoxyribonucleic acid synthesis by phenethyl alcohol. J. Bacteriol. 1964, 88, 1580-1584.

31. Anbazhagan, V.; Munz, C.; Tome, L.; Schneider, D. Fluidizing the membrane by a local anesthetic: Phenylethanol affects membrane protein oligomerization. J. Mol. Biol. 2010, 404, 773-777.

32. Chen, L.; Tai, P.C. Effects of antibiotics and other inhibitors on ATP-dependent protein translocation into membrane vesicles. J. Bacteriol. 1987, 169, 2373-2379.

33. Greene-McDowelle, D.M.; Ingber, B.; Wright, M.S.; Zeringue, H.J., Jr.; Bhatnagar, D.; Cleveland, T.E. The effects of selected cotton-leaf volatiles on growth, development and aflatoxin production of Aspergillus parasiticus. Toxicon 1999, 37, 883-893.

34. Wright, M.S.; Greene-McDowelle, D.M.; Zeringue, H.J.; Bhatnagar, D.; Cleveland, T.E. Effects of volatile aldehydes from Aspergillus-resistant varieties of corn on Aspergillus parasiticus growth and aflatoxin biosynthesis. Toxicon 2000, 38, 1215-1223.

35. Cleveland, T.E.; Carter-Wientjes, C.H.; de Lucca, A.J.; Boue, S.M. Effect of soybean volatile compounds on Aspergillus flavus growth and aflatoxin production. J. Food. Sci. 2009, 74, H83-H87.

36. Chang, P.-K.; Scharfenstein, L.L.; Mack, B.; Yu, J.; Ehrlich, K.C. Transcriptomic profiles of Aspergillus flavus CA42, a strain that produces small sclerotia, by decanal treatment and after recovery. Fungal Genet. Biol. 2014, 68, 39-47.

37. White, B.T.; Yanofsky, C. Structural characterization and expression analysis of the Neurospora conidiation gene con-6. Dev. Biol. 1993, 160, 254-264. 
38. Adams, T.H.; Hide, W.A.; Yager, L.N.; Lee, B.N. Isolation of a gene required for programmed initiation of development by Aspergillus nidulans. Mol. Cell Biol. 1992, 12, 3827-3833.

39. Wieser, J.; Lee, B.N.; Fondon, J., 3rd; Adams, T.H. Genetic requirements for initiating asexual development in Aspergillus nidulans. Curr. Genet. 1994, 27, 62-69.

40. Chang, P.-K.; Scharfenstein, L.L.; Mack, B.; Ehrlich, K.C. Deletion of the Aspergillus flavus orthologue of $A$. nidulans $f l u G$ reduces conidiation and promotes production of sclerotia but does not abolish aflatoxin biosynthesis. Appl. Environ. Microbiol. 2012, 78, 7557-7563.

41. Keller, N.P.; Turner, G.; Bennett, J.W. Fungal secondary metabolism-From biochemistry to genomics. Nat. Rev. Microbiol. 2005, 3, 937-947.

42. Li, R.W.; Rinaldi, M.; Capuco, A.V. Characterization of the abomasal transcriptome for mechanisms of resistance to gastrointestinal nematodes in cattle. Vet. Res. 2011, 42, 114.

43. CLC bio, a Qiagen Company. Available online: http://www.clcbio.com (accessed on 22 September 2015).

44. Aspergillus flavus, Genome, NCBI. Available online: http://www.ncbi.nlm.nih.gov/genome/ ?term=aspergillus + flavus (accessed on 22 September 2015).

45. Mortazav, A.; Williams, B.A.; McCue, K.; Schaeffer, L.; Word, B. Mapping and quantifying mammalian transcriptomes by RNA-Seq. Nat. Methods 2008, 7, 621-628.

46. Robinson, M.D.; Smyth, G.K. Small-sample estimation of negative binomial dispersion, with applications to SAGE data. Biostatistics Oxford Engl. 2008, 9, 321-332.

47. Benjamini, Y.; Hochberg, Y. Controlling the false discovery rate: A practical and powerful approach to multiple testing. J. R. Stat. Soc. Ser. B 1995, 57, 289-300.

48. Conesa, A.; Götz, S. Blast2GO: A comprehensive suite for functional analysis in plant genomics. Int. J. Plant Genom. 2008, 2008, 619832.

49. Kanehisa, M.; Goto, S.; Sato, Y.; Furumichi, M.; Tanabe, M. KEGG for integration and interpretation of large-scale molecular data sets. Nucleic Acids Res. 2012, 40, D109-D114.

(C) 2015 by the authors; licensee MDPI, Basel, Switzerland. This article is an open access article distributed under the terms and conditions of the Creative Commons Attribution license (http://creativecommons.org/licenses/by/4.0/). 